\title{
Comprehensive cardiac rehabilitation programme for implantable cardioverter-defibrillator patients: a randomised controlled trial
}

\author{
A Fitchet, P J Doherty, C Bundy, W Bell, A P Fitzpatrick, C J Garratt
}

Heart 2003;89:155-160

See end of article for authors' affiliations ........................

Correspondence to: Dr A Fitchet, Manchester Heart Centre, Manchester Royal Infirmary, Oxford Road, Manchester M13 9WL, UK; alan.fitchet@talk21.com

Accepted 18 September 2002

\begin{abstract}
Objective: To investigate the effects of a 12 week comprehensive cardiac rehabilitation (CCR) programme on patients who have undergone implantation of an implantable cardioverter-defibrillator (ICD).

Design: Sixteen patients with ICDs (14 (88\%) male, mean (SD) age 58 (10) years, range 34-74 years) were randomised to either attend an individually tailored CCR programme or receive usual care. They then changed to the alternative regimen for a further 12 weeks. Exercise capacity was assessed using a treadmill exercise test at baseline, after usual care, after CCR and 12 weeks after CCR to assess maintenance effects. Hospital anxiety and depression (HAD) scores were recorded at each stage. Results: Exercise times (min:s; mean (SD)) increased by 16\% from a baseline mean of 9:55 (2:33) to $11: 11$ (2:17) following attendance at CCR (95\% confidence interval (C) $0: 34$ to $1: 58 ; p=0.001)$. This improvement was maintained 12 weeks after attendance at CCR, at 11:20 $(2: 17)(p=1.00)$. HAD scores for anxiety and depression decreased during CCR from a baseline of $13.4(3.6)$ to 8.1 (3.6), $95 \% \mathrm{Cl} 3.5$ to $7.0(\mathrm{p}<0.001$ ) and $9.9(3.4)$ to $6.7(2.9), 95 \% \mathrm{Cl} 1.9$ to $4.4(\mathrm{p}=0.002)$, respectively. These improvements were maintained at 12 weeks after CCR. No ventricular arrhythmias or ICD discharges occurred during the exercise components of the CCR. The total number of ventricular arrhythmias and ICD discharges was similar 12 weeks before, during, and 12 weeks after CCR. Conclusions: CCR appears to be safe for patients with ICDs. It can improve exercising ability and lower the levels of psychological distress. A larger multicentre study is recommended to confirm these findings.
\end{abstract}

$\mathrm{T}$ he implantable cardioverter-defibrillator (ICD) is highly effective in the management of life threatening ventricular arrhythmias, with benefits shown in both primary and secondary prevention studies. ${ }^{1-4}$ The National Institute for Clinical Excellence has recommended a major increase in their use in the UK. It also recommended "a rehabilitative approach to aftercare, which includes psychological preparation for living with an ICD" . ${ }^{5}$ Little work has been done on the psychosocial sequelae of life threatening arrhythmias and subsequent ICD implantation in the UK. In our experience some patients fail to adapt to these traumatic events, developing phobic anxiety states, depression, and a fear of exercise lest their ICDs discharge. Dubin and colleagues reported that $63 \%$ of a cohort of ICD patients aged below 40 years were worried about engaging in exercise.

Cardiac rehabilitation is established for patients with ischaemic heart disease with reported benefits for exercising ability, psychological functioning, and prognosis. ${ }^{7}$ Patients with chronic heart failure show improvements in exercise performance and autonomic function with exercise training alone. ${ }^{89}$ Several groups have suggested that patients with ICDs may also benefit from structured rehabilitation, including exercise training, but the effects of this have not been studied. ${ }^{10-12}$

The British Association for Cardiac Rehabilitation, consistent with other major cardiac rehabilitation organisations, recommends the use of "comprehensive" cardiac rehabilitation (CCR), the key components of which are exercise, education, and psychological support. ${ }^{13-15}$ We designed such a programme specifically for ICD patients and have assessed its effects in a randomised controlled prospective study.

\section{METHODS}

Patients

All 73 of the patients consecutively implanted with ICDs under the care of the Manchester Heart Centre were sent a request that they should participate in the CCR programme. Fifty nine $(81 \%)$ of these were male and $14(19 \%)$ female. Mean (SD) age was 57 (14) years (range 14-79 years) and their left ventricular ejection fraction was 41 (19)\% (range $5-70 \%)$. Thirty four patients agreed to take part and 16 of these were randomly selected to attend the programme. All 16 subsequently consented to take part in the study. Their characteristics were similar: $14(88 \%)$ were male, their mean age was 58 (10) years (range 34-74 years), and their left ventricular ejection fraction was 38 (17)\% (range 12-70\%). In eight patients $(50 \%)$ the ejection fraction was below $35 \%$.

Underlying cardiac diagnoses were ischaemic heart disease (11), dilated cardiomyopathy (2), valvar heart disease (1), arrhythmogenic right ventricular cardiomyopathy (1), and Brugada syndrome (1). One patient was in atrial fibrillation, but all the others were in sinus rhythm. ICDs had been implanted for a mean of 20.4 (13.8) months (range 7-53 months). All ICDs were single chamber devices. Five patients were taking amiodarone, nine were on $\beta$ blockers, nine were on angiotensin converting enzyme (ACE) inhibitors, and five were taking diuretics. One patient was on digoxin, one was taking losartan, and two were taking other antianginal drugs (table 1).

Abbreviations: $C C R$, comprehensive cardiac rehabilitation; $H A D$ hospital anxiety and depression score; ICD, implantable cardioverter-defibrillator; RPE, rate of perceived exertion 


\begin{tabular}{|c|c|c|c|c|c|c|}
\hline Patient & Group & Age (years) & $\beta$ Blocker & ACE inhibitor & Other & Notes \\
\hline 1 & A & 48 & & L 10 & Amiodarone, statin & B 2.5 added post-CCR \\
\hline $2^{*}$ & A & 64 & B 10 & & Statin & \\
\hline 3 & A & 62 & & & ISMO, statin & \\
\hline 4 & A & 66 & A 100 & L 5 & ISMO, diltiazem, statin & Nicorandil added at start of CCR \\
\hline 5 & A & 55 & & E 2.5 & Statin & Frumil added post-CCR \\
\hline $6 \dagger$ & A & 63 & M 50 & L 10 & Amiodarone & \\
\hline $7^{*}$ & A & 54 & C 12.5 & L 10 & Digoxin & \\
\hline $8^{*}$ & A & 43 & S 40 & & & \\
\hline 1 & B & 52 & & L 20 & Amiodarone, frusemide & M 25 added at start of CCR \\
\hline 2 & B & 71 & A 25 & & Statin & \\
\hline 3 & B & 74 & B 5 & L 10 & Bumetanide & \\
\hline 4 & B & 59 & A 50 & & & \\
\hline $5^{*}$ & B & 59 & & & Amiodarone, bumetanide, losartan & \\
\hline 6 & B & 67 & & L 10 & Amiodarone, bumetanide, statin & \\
\hline 7 & B & 62 & & L 10 & Frusemide & \\
\hline 8 & B & 34 & S 240 & $\mathrm{Ca} 12$ & & \\
\hline
\end{tabular}

*Did not attend/complete CCR.

†Did not complete 12 week post-CCR exercise test.

Numbers refer to daily doses in $\mathrm{mg}$.

A, atenolol; B, bisoprolol; C, carvedilol; Ca, captopril; CCF, congestive caerdiac failure; CCR, comprehensive cardiac rehabilitation; E, enalapril; Frumil, frusemide + amiloride; ISMO, isosorbide mononitrate; L, lisinopril; M, metoprolol; S, sotalol.

\section{Design}

A randomised controlled prospective study was designed to investigate the influence of attendance at a 12 week CCR programme on exercise capacity and psychosocial functioning. The 16 patients were randomised to two groups ( $\mathrm{A}$ and $\mathrm{B}$ ) to either attend the CCR programme or receive usual care for 12 weeks. They then changed to the alternative regimen for a further 12 weeks. Physical, cardiological, and psychosocial variables were measured at baseline, before starting CCR (in group A), at the end of CCR, and 12 weeks after CCR to assess maintenance effects (fig 1). Throughout the study period patients had access to a dedicated 24 hour ICD advice help line manned by an electrophysiological specialist nurse, ready access to technicians, and a well attended ICD patient support group.

Exclusion criteria were physical infirmity precluding exercise, New York Heart Association (NYHA) functional class IV heart failure or angina, and an inability to give informed consent. No patient who requested to take part was excluded before the start of the study.

Outcome measures recorded were change in exercise test time, frequency of ICD discharges, frequency of ventricular arrhythmias, and change in HAD scale scores for anxiety and depression.

\section{Comprehensive cardiac rehabilitation programme}

Patients attended the CCR programme for two hours twice a week. It combined an individually prescribed aerobic exercise programme with education and a cognitive behavioural intervention. Supplementary exercise in the community was encouraged between sessions. Spouses were encouraged to attend as well, and approximately $50 \%$ did so on a regular basis.

\section{Exercise testing}

Exercise tolerance was assessed using a symptom limited treadmill exercise test with a continuous 12 lead ECG and regular blood pressure monitoring.

A modified Kattus protocol ${ }^{16}$ was used, starting at $1.6 \mathrm{~km} / \mathrm{h}$ ( $1.0 \mathrm{mph}$ ) with increments of $0.8 \mathrm{~km} / \mathrm{h}(0.5 \mathrm{mph})$ every two minutes up to a maximum of $7.2 \mathrm{~km} / \mathrm{h}(4.5 \mathrm{mph})$ at a constant gradient of $10 \%$. The incremental stages were shortened from three to two minutes in order to increase the workload more rapidly and reduce the likelihood of patient fatigue becoming the test end point. It was intended to determine the patients' ability to perform aerobic exercise ideally for a minimum of six minutes ${ }^{17}$ in order to plan their exercise regimens.
The physiological demands of the exercise test were expressed and monitored in a relative context using a rate of perceived exertion (RPE) scale. ${ }^{18}$ This scale ranged from 0 to 10, 0 being "no effort" through 5, "moderate effort," to 10 , "maximum effort." The RPE scale subsequently allowed self monitoring of exercise throughout the programme.

Exercise was terminated when one of the following three end points was reached:

- an RPE scale of 7/10 or "hard effort"17

- heart rate of $75 \%$ of the age adjusted maximum ${ }^{17}$

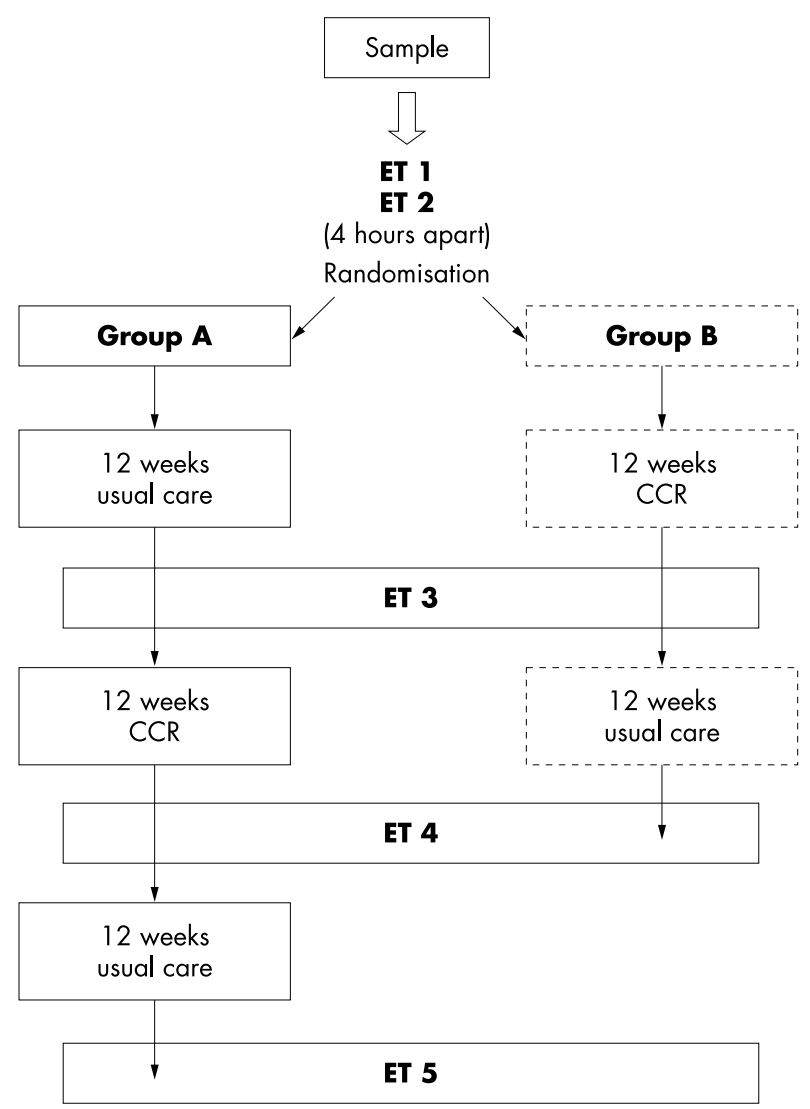

Figure 1 Study design. CCR, comprehensive cardiac rehabilitation; ET, exercise test. 
- heart rate of 10 beats below ICD activation threshold; this was deemed sufficient to accommodate the small additional rise in heart rate that may occur following achievement of peak exercise.

A graduated "cool down" period followed peak exercise, with exercise being terminated abruptly only if the patient or clinical circumstances dictated. This was designed to reduce ventricular arrhythmias in recovery and is discussed later.

Exercise tests were directed by a specialist physiotherapist and a physician experienced in the management of patients with ICDs. Full resuscitation facilities were available. ICDs were interrogated before the exercise to confirm detection parameters and download stored information on arrhythmias and device activity.

At initial assessment all patients underwent two exercise tests-prebaseline and baseline-four hours apart. This helped allay initial fears of exercise and allowed familiarity with the treadmill and monitoring equipment, with the aim of defining a true baseline. Each patient's second test formed their baseline.

\section{Exercise prescription}

Exercise test intensity, heart rate, and RPE were used to shape individual exercise programmes. The effective training zone was based on a heart rate range from $60-75 \%$ of the age adjusted maximum, ${ }^{17}$ with an absolute upper limit set at 10 beats below ICD activation threshold. The programme consisted of an initial familiarisation phase followed by an aerobic improvement phase and finally maintenance. Nine exercises were used, each with four levels (table 2). Each level was separated by approximately two metabolic equivalents (METs), with the first level set at 3-4 METs. Exercises were performed standing. Seated exercises were avoided in an attempt to minimise the adverse impact of poor ventricular function. ${ }^{10}$

Supervised exercise was performed at each session, with supplementary exercise encouraged at home. Telemetry wrist monitors (Polar Electro Oy, Kempele, Finland) were provided throughout the initial exercise phase in order to facilitate feedback and aid compliance with advised training heart rates.

\section{Education}

Healthcare professionals chaired informal 30 minute seminars at alternate CCR sessions. Subjects covered included benefits of exercise, cardiovascular disease, ICD technology, and diet. Open group discussion filled the remaining sessions

\section{Psychology}

Psychological distress was measured throughout the study using the hospital anxiety and depression (HAD) scale. ${ }^{19} \mathrm{~A}$ health psychologist with experience of cardiac patients provided psychological input. This comprised three main components:

Table 2 Exercise class content: the exercise circuit had nine exercises with four levels for each; exercises were performed standing

- Shuttle walk

- Lift ball above head and down

- Stepping on the spot

- Ball around the body

- Alternate leg: step and lunge

- Squats

- Stepping out: side to side

- Step ups (to metronome)

- Arm lifts (can be done while stepping)
- a cognitive-behavioural framework that addressed the link between thoughts, feelings, and behaviour regarding ICDs and illness

- a group session that addressed management of anxiety, anger, and depression; patients who scored in the clinical range for anxiety, depression, or excessive anger received individual psychological support

- self help strategies: these included progressive muscular relaxation, rebreathing techniques, deep relaxation, and self hypnosis.

\section{Statistical analysis}

Comparisons were made at the following points in the protocol: at baseline, before CCR, at the end of CCR, and 12 weeks after CCR. All data were subjected to repeated measures analysis of variance (ANOVA).

\section{RESULTS}

All 16 patients performed the two baseline exercise tests. One patient (5B), who was unable to cope with the modified Kattus protocol because of difficulty in walking at the required speed and gradient, was exercised at a speed of $1.0 \mathrm{~km} / \mathrm{h}$ (0.6 mph) at zero gradient.

Two patients (2A and $8 \mathrm{~A}$ ) reported, after randomisation, that they could not attend the CCR programme because of financial difficulties with travelling to the hospital. A further patient (7A), who lived alone, attended only two CCR sessions because a short term memory deficit prevented him remembering appointments and the recommended exercise regimen. One patient (5B) completed the CCR programme but developed progressive heart failure latterly and was excluded from further participation in the study. Finally, one patient (6A) was unable to perform the end of the maintenance period exercise test because of an infective exacerbation of chronic airways disease. Thus 13 patients in all completed the CCR programme and the post-CCR exercise test, with 11 patients completing all exercise tests.

There was no difference in age, left ventricular function, baseline exercise time, or HAD scores between the patients who completed the CCR and those who failed to attend.

No patients who attended CCR suffered ICD discharges during either exercise testing, supervised exercise, or exercise at home. The total number of ICD discharges during the study period was 2, which occurred in two different patients, one of whom (5B) developed heart failure towards the end of the CCR programme. This compared with no ICD discharges during the three months before rehabilitation and two (in two patients) during the three months following the programme. Sustained ventricular tachycardia successfully terminated by anti-tachycardia pacing occurred three times (in two patients) during the CCR, compared with twice (in two patients) during the three months preceding rehabilitation, and twice (in two patients) during the three months following the programme. Non-sustained ventricular tachycardia not requiring ICD treatment occurred 18 times (in two patients) during the CCR, compared with 20 times (in one patient) during the three months preceding rehabilitation and 22 times (in four patients) during the three months following the programme.

\section{Exercise test times}

The first and second exercise tests were designated "prebaseline" and "baseline" tests. Exercise time (min:s; mean (SD)) increased as a group from a prebaseline value of 8:12 (3:24) to a baseline value of 9:16 (3:04), a mean increase of 13\% (95\% confidence interval (CI) $0: 26$ to $1: 41 ; p=0.002$ ). The standard error of measurement of the paired differences between these tests was 48 seconds. This determined a range within which there was a $95 \%$ probability that measurements would lie. Using this range as a cut off, eight of the 16 patients achieved 


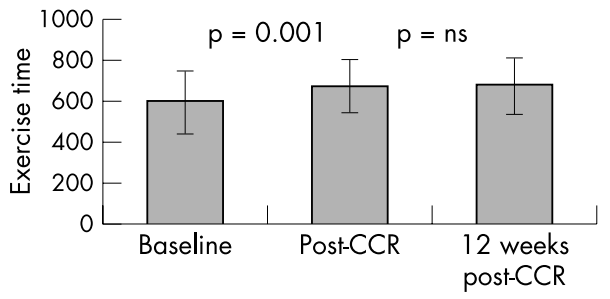

Figure 2 Exercise times: baseline, immediately after comprehensive cardiac rehabilitation (CCR), and 12 weeks after CCR. Values are mean times (s) for the 11 patients who completed the CCR and all the exercise tests. Error bars $=S D$

a significant improvement in exercise time, seven achieved a similar time, and one patient $(7 \mathrm{~B})$ achieved a reduced time in the second exercise test compared with the first. Patient 7B was a regular treadmill user and his second test, in contrast to the majority of patients, may have been influenced more by fatigue than by learning effect.

Repeated measures ANOVA analysis of the 11 patients who completed all exercise tests (all male, mean (SD) age 59 (11) years, range 34-74 years; left ventricular ejection fraction 39 (14)\%, range $20-65 \%$ ) showed that mean exercise time (min:s) increased by $16 \%$, from a baseline of 9:55 (2:33) to 11:11 (2:17) following attendance at CCR (95\% CI 0:34 to $1: 58 ; \mathrm{p}=0.001)$. This improvement in exercise time was maintained at 12 weeks after the end of the CCR, at 11:20 (2:17) (fig 2).

The eight patients in group A received usual care for 12 weeks following baseline tests before starting the CCR programme, thus providing a control group. A further exercise test was done immediately before starting the CCR. There was no difference between exercise test times at baseline (9:53 (2:50) min:s, range 5:31 to 13:11) and following the 12 week control period $(9: 54$ (3:14) min:s, range $4: 22$ to $13: 00)$ $(p=0.96)$. The increase in exercise times between the prebaseline and baseline tests, without further increase during the 12 week control period, validated the requirement of a second exercise test to define a true baseline.

\section{Psychology}

Patients completed HAD questionnaires at each stage of the study. This is a well validated and reliable scale used extensively in the cardiac population. ${ }^{19}$ A score of 11 or more indicates clinically significant anxiety or depression.

\section{Anxiety scores}

Mean (SD) baseline anxiety scores were 10.8 (4.6) in group A, 13.5 (3.3) in group B, and 12.1 (4.1) in both groups combined. During the control period, anxiety scores rose to 13.3 (2.5), $95 \%$ CI 0.35 to $4.6(p=0.028)$ in group A. Significant levels of anxiety were present in four patients in group A, seven in group B, and 11 in both groups combined at baseline. During the control period seven patients in group A demonstrated significant anxiety.

Further analysis is confined to the 11 patients who completed the CCR and all the exercise tests. The baseline anxiety score in these patients was 13.4 (3.6). This decreased during CCR to $8.1(3.6), 95 \%$ CI 3.5 to 7.0 (p $<0.001)$ and was then maintained at $6.1(4.5)(\mathrm{p}=0.60)$ at 12 weeks after CCR. Significant levels of anxiety were present in nine of the 11 patients before CCR, reducing to four at the end of CCR, and to two at 12 weeks after CCR (fig 3).

\section{Depression scores}

Mean (SD) baseline depression scores were 7.6 (4.5) in group A, 10.1 (3.1) in group B, and 8.9 (3.9) in both groups combined. During the control period, depression scores did not change in group A: 9.5 (3.2), 95\% CI 0.24 to 4.0

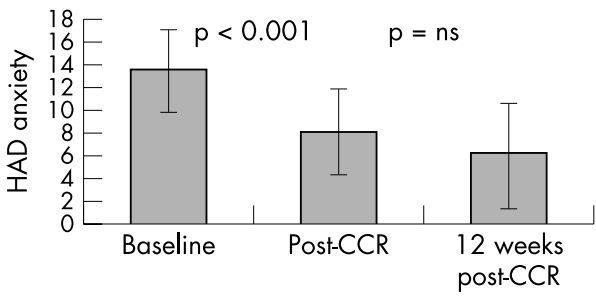

Figure 3 Hospital anxiety and depression (HAD) scores for anxiety. Values are means for the 11 patients who completed the comprehensive cardiac rehabilitation (CCR) and all the exercise tests. Error bars $=S D$.

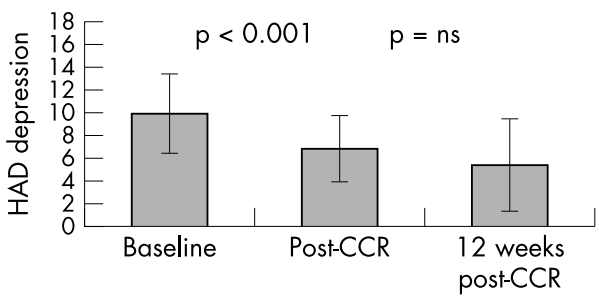

Figure 4 Hospital anxiety and depression (HAD) scores for depression. Values are means for the 11 patients who completed the comprehensive cardiac rehabilitation (CCR) and all the exercise tests. Error bars $=\mathrm{SD}$.

$(p=0.074)$. Significant levels of depression were present in two patients in group A, five in group B, and seven in both groups combined at baseline. During the control period three patients in group A demonstrated significant depression.

Further analysis is confined to the 11 patients who completed the CCR and all the exercise tests. The baseline depression score in these patients was 9.9 (3.4). This decreased during CCR to $6.7(2.9), 95 \%$ CI 1.9 to 4.4 $(p<0.001)$ and was maintained at $5.5(3.9)(p=0.75)$ at 12 weeks after CCR. Significant levels of depression were present in six patients before CCR, reducing to two at the end of CCR, and further to one at 12 weeks after CCR (fig 4).

\section{CCR appraisal}

All 13 patients who completed the CCR also completed a simple anonymous postal CCR appraisal questionnaire. Ten patients $(77 \%)$ reported that attendance at the CCR had been "very beneficial", the remainder reporting "some benefit". Ten patients also reported that the CCR had made them "feel more positive" about living with their ICD, the remainder reporting "no change". Eleven patients $(85 \%)$ reported that they felt more confident in taking exercise, the remainder reporting no change. All participants stated that the exercise component of the CCR was among the most helpful.

\section{DISCUSSION}

The main findings of this study are that comprehensive cardiac rehabilitation of patients with ICDs produces improvement in exercising ability and reduces levels of anxiety and depression.

\section{Exercise training}

Patients with ICDs comprise one group that has not been offered comprehensive rehabilitation to date. Indeed patients with a history of ventricular arrhythmias have been excluded from the exercise components of CCR programmes because of safety concerns. However, most published work to date concerns maximal exercise tests in order to assess ischaemic heart disease burden, predisposition to arrhythmias, or efficacy of antiarrhythmic drug treatment. Significant rates of ventricular arrhythmias have resulted. Young and colleagues exercised 263 patients with a history of malignant ventricular 
arrhythmias, in a total of 1377 maximal exercise tests. Arrhythmias requiring medical attention occurred in $9.1 \%$ of patients during $2.3 \%$ of tests, compared with $0.12 \%$ of patients during $0.05 \%$ of tests in a reference population. The arrhythmias were ventricular fibrillation in $28 \%$, ventricular tachycardia in $69 \%$, and bradycardia in $3 \%{ }^{20}$ The aim of our CCR was one of exercise training and not diagnosis. The prescribed exercise was aerobic and individually tailored in order to avoid the extra adrenergic load and predisposition to arrhythmias that maximal exercise would produce.

A small amount of work has been published on exercising patients with ICDs. Friedman and colleagues reported cases of two ICD patients benefiting physically and psychologically from attendance at a cardiac rehabilitation programme. ${ }^{11}$ They recommended deactivation of the device during exercise testing. Lampman and colleagues also recommended deactivation of the ICD during baseline exercise testing before prescribing exercise. ${ }^{10}$ In contrast, we believe that all exercise should be performed with the ICD activated in order to reinforce positive feedback that such exercise is safe and can be continued away from the controlled environment of the CCR programme.

A graduated "cool down" period was built into each patient's exercise tests, with exercise being terminated abruptly only if clinical circumstances dictated. Cool down was also implemented during supervised exercise and encouraged when exercising in the community. Pashkow and colleagues reported an increased risk of malignant ventricular arrhythmias in the early recovery period after exercise testing. ${ }^{21}$ This may result from the surge in circulating concentrations of catecholamines, particularly noradrenaline (norepinephrine) measured at this time..$^{22}$ A gradual "cool down" rather than abrupt cessation of exercise may minimise this surge, offering a degree of protection to susceptible individuals. Observational evidence for this is provided by a survey of 71914 maximal exercise tests performed at a single medical centre between 1971 and $1987 .{ }^{23}$ Six major cardiac complications, including one death, occurred in the period before 1979 , following which a "cool down" period of three minutes for normal tests and five minutes for abnormal tests was introduced. No complications occurred in the ensuing 45000 tests over the next 10 year period.

Significant improvements in exercise time were seen following attendance at the CCR programme and were maintained for at least 12 weeks afterwards. It is likely that improvements in both physical and psychological status provided positive feedback to enhance one another and increase exercising ability. An improvement in cardiorespiratory function was suggested by the increases in exercise time seen without increase in heart rate or rate of perceived exertion. However, as neither cardiovascular nor ventilatory function was formally assessed, their contribution to the reduction of anxiety resulting from physical training is unclear. When formally measured, improvements in cardiorespiratory function and exercise time have been demonstrated following maximal exercise training in patients with controlled heart failure. ${ }^{24}$ Our study suggests that such benefits may be achieved with submaximal exercise training.

The exercise testing component of the CCR was labour intensive, requiring a technician, a specialist physiotherapist, and a cardiologist. The reassuring safety record of the 64 tests performed in this study suggests that a physician may not need to be physically present but could be readily available nearby. Exercise tests were essential in planning exercise regimens and appeared to provide reliable exercise times on the second test in this group. It is possible that patients who are familiar with treadmill testing would require a single test alone. CCR classes were limited to eight in this study to maintain a ratio of four patients to each member of staff.

\section{Psychological benefits}

Increased levels of anxiety and depression have been described in between $20-58 \%$ of patients after ICD implantation. ${ }^{25-29}$ Florid psychopathology requiring psychiatric referral was reported in six of 35 patients $(17 \%)$ by Bourke and colleagues. ${ }^{30}$ Similar levels of psychological distress were found in our study group. Among those who completed the CCR the prevalence of anxiety or depressive states at baseline was as high as $67 \%$. This decreased to $42 \%$ at the end of CCR and to $25 \%$ at 12 weeks after CCR, suggesting that psychological benefits were maintained for at least 12 weeks. It is likely that reductions in psychological distress played a significant role in the patients' enhanced functional status. Such reductions probably reflected a combination of positive feedback resulting from an enhanced ability to exercise and psychological support/intervention. Kohn and colleagues reported a reduction in trait anxiety and major depressive episodes with cognitive behavioural therapy alone after ICD implants. ${ }^{31}$

\section{Benefits of support groups}

Following ICD implantation, many patients report subjective benefit from attendance at patient support groups, but objective evidence of this is lacking. Differences in psychological scoring between support group attendees and controls were not found in studies by Badger and Morris, ${ }^{32}$ Molchany and Peterson, ${ }^{33}$ or Sneed and colleagues. ${ }^{34}$ In our study, patients already had access to an active support group and a 24 hour telephone help line, yet still gained psychologically from participation in the CCR programme. This suggests that psychological benefits gained from structured CCR are above and beyond those from supportive therapy alone.

\section{Travelling to rehabilitation programmes}

This CCR programme was based in a tertiary referral centre serving the north western area of England, which necessitated patients travelling distances of up to 100 miles each way to attend. This caused financial difficulties for two, with failure to attend, and possibly dissuaded others from volunteering to take part. Debarment from driving for at least six months after ICD implantation potentially caused further travelling difficulties for many. Encouraging spouses/partners also to attend alleviates some of these difficulties. It is likely that as the use of ICDs expands they will increasingly be implanted in non-tertiary hospitals, and "satellite" support facilities and rehabilitation programmes will be developed in district settings. This is likely to ease some of the travel problems.

\section{Study limitations}

Our study investigated the effects of the CCR programme on the first two cohorts of patients to participate. They were under regular cardiological follow up and their drug treatment was optimised before they entered the study. In spite of this, the diversity and potential instability of their cardiac conditions did not allow control for either the drugs used or any changes in treatment occurring during the programme. Metoprolol was introduced in patient $1 \mathrm{~B}$ at the beginning of the CCR following an episode of ventricular tachycardia. Bisoprolol was introduced in patient $\mathrm{IA}$ at the end of the CCR for symptomatic control of new onset hyperthyroidism. In patient $4 \mathrm{~A}$, lisinopril was increased from $5 \mathrm{mg}$ to $10 \mathrm{mg}$ during the usual care control phase and then reduced back to $5 \mathrm{mg}$ with the addition of nicorandil after CCR. Frumil (frusemide plus amiloride) was added to the treatment regimen of patient $5 \mathrm{~A}$ during the usual care control phase following an episode of breathlessness.

Our cohort of patients was small and had an important drop out rate. Thirteen patients ( $81 \%$ ) completed CCR and a full set of data was available in $11(69 \%)$. It was not possible to obtain complete follow up data on the patients who dropped out, so analysis was based on those who completed the CCR and all of the outcome assessments, rather than on an intention to treat basis. The lack of difference in baseline characteristics 
between those who completed the CCR and those who failed to attend supports such an analysis.

In spite of an acceptance rate of $62 \%$ for participation in the CCR, there remains a possibility of selection bias. Those who volunteered may represent individuals with a higher initial morbidity, greater anxiety levels, greater enthusiasm to improve, and most to gain from structured rehabilitation. The voluntary nature of the selection may also have biased against inclusion of patients with greater severity of depression. Such bias could be reduced or eliminated by a larger study and by prospectively enrolling consecutive patients at a fixed datefor example, three months after ICD implantation. Our patients reported psychological distress at a mean of 20.4 (range 7-53) months post ICD implant. It is possible that if CCR were performed earlier after ICD implantation, psychological distress and maladaptive behaviour could be supported and prevented from development.

\section{Conclusions}

The combination of a safe exercise regimen, education to challenge misconceptions about exercise, and the development of alternative coping strategies was successful in improving psychological health and the ability to exercise in a group of patients with ICDs. This was achieved in a relatively short intervention. This small study suggests that structured comprehensive rehabilitation may be beneficial to patients with ICDs. A larger prospective multicentre study should be done to confirm these findings.

\section{ACKNOWLEDGEMENT}

The rehabilitation programme was supported by a grant from the Britsh Heart Foundation. We thank Bruno Rushforth, fourth year psychology student, for his help in collation and analysis of the psychology data.

\section{Authors' affiliations}

A Fitchet, W Bell, A P Fitzpatrick, C J Garratt, Manchester Heart Centre, Manchester Royal Infirmary, Manchester, UK

P J Doherty, School of Physiotherapy, Manchester Royal Infirmary

C Bundy, School of Medicine, University of Manchester

\section{REFERENCES}

1 Moss AJ, Jackson Hall W, Cannom DS, et al for the Multicenter Automatic Defibrillator Implantation Trial Investigators. Improved surviva with an implanted defibrillator in patients with coronary disease at high risk for ventricular arrhythmia. N Engl J Med 1996;335:1933-40.

2 The AVID investigators. A comparison of antiarrythmic-drug therapy with implantable defibrillators in patients resuscitated from near-fatal ventricular arrhythmias. N Engl J Med 1997;337:1576-83.

3 Siebels J, Kuck KH. Implantable cardiac defibrillator compared with antiarrhythmic drug treatment in cardiac arrest survivors (the Cardiac Arrest Study Hamburg). Am Heart J 1994;127:1 139-44.

4 Buxton $\mathrm{AE}$, Lee $\mathrm{KL}$, Fisher JD, et al. A randomised study of the prevention of sudden death in patients with coronary artery disease. Multicenter unsustained tachycardia trial investigators. N Engl J Med 1999;341:1882-90.

5 National Institute for Clinical Excellence. Guidance on the use of implantable cardioverter defibrillators for arrhythmias. NICE technology appraisal guidance, No 11. London: NICE, 2000

6 Dubin AM, Batsford WP, Lewis R, et al. Quality of life in patients receiving implantable cardioverter defibrillators at or before age 40 . PACE 1996;19:1555-9.
7 Ades PA, Coello CE. Effects of exercise and cardiac rehabilitation on cardiovascular outcomes. Med Clin North Am 2000;84:251-65.

8 Coats AJ, Adamopoulos S, Radaelli A, et al. Controlled trial of physical training in chronic heart failure. Exercise performance, haemodynamics, ventilation, and autonomic function. Circulation 1992;85:2119-31.

9 European Heart Failure Group. Experience from controlled trials of physical training in chronic heart failure. Protocol and patient factors in effectiveness in the improvement in exercise tolerance. Eur Heart J 1998; 19:466-75

10 Lampman RM, Knight BP. Prescribing exercise training for patients with defibrillators. Am J Phys Med Rehabil 2000;79:292-97.

11 Friedman AW, Lipman RC, Silver SJ, et al. Cardiac rehabilitation/ exercise in patients with implantable cardioverter defibrillators. J Natl Med Assoc 1996;88:374-8.

12 Lewin RJP, Frizelle DJ, Kaye GC. A rehabilitive approach to patients with internal cardioverter-defibrillators. Heart 2001;85:371-2.

13 Coats AJ, McGee H, Stokes H, et al. BACR guidelines for cardiac rehabilitation. Oxford: Blackwell Science, 1995.

14 Stone JA, Cyr C, Friesen M, et al. Canadian guidelines for cardiac rehabilitation and atherosclerotic disease prevention: a summary. Can J Cardiol 2001;17(suppl B):3B-30B.

15 Balady GJ, Ades PA, Comoss P, et al. Core components of cardiac rehabilitation/secondary prevention programmes. A statement for healthcare professionals from the American Heart Association and the American Association of Cardiovascular and Pulmonary Rehabilitation. Circulation 2000; 102:1069-73

16 Kattus A, Alvano A, MacAlpine R. Treadmill exercise tests for capacity and adaptation in angina pectoris. J Occup Med 1968;10:627-35.

17 American College of Sports Medicine. Guidelines for exercise testing and prescription, 6th ed. Baltimore: Williams and Wilkins, 2000; 185-93.

18 Borg GA. Psychophysical bases of perceived exertion. Med Sci Sports Exerc 1982;14:317-81

19 Zigmond AS, Snaith RP. The hospital anxiety and depression scale. Acta Psychiatr Scand 1983;67:361-70.

20 Young DZ, Lampert S, Graboys TB, et al. Safety of maximal exercise testing in patients at high risk for ventricular arrhythmia. Circulation 1984;70:184-91.

21 Pashkow FJ, Schweikert RA, Wilkoff BL. Exercise testing and training in patients with malignant arrhythmias. Exerc Sport Sci Rev 1997;25:235-69.

22 Dimsdale JE, Hartley LH Guiney T, et al. Postexercise peril: plasma catecholamines and exercise. JAMA 1984;251:630-63.

23 Gibbons L, Blair SN, Kohl HW, et al. The safety of maximal exercise testing. Circulation 1989;80:846-52

24 Wielenga RP, Coats AJS, Mosterd WL, et al. The role of exercise training in chronic heart failure. Heart 1997;78:431-6.

25 Hegel MT, Griegel LE, Black C, et al. Anxiety and depression in patients receiving implanted cardioverter-defibrillators: a longitudinal investigation. Int J Psychiatry Med 1997;27:57-69.

26 Heller SS, Ormont AM, Lidagoster L, et al. Psychosocial outcome after ICD implantation: a current perspective. PACE 1997;21:1207-15

27 Pycha C, Calabrese JR Gulledge, et al. Patient and spouse acceptance and adaptation to implantable cardioverter defibrillators. Cleve Clin J Med 1992:57:441-4

28 Schuster PH, Philips S, Dillons DL, et al. The psychosocial and physiological experiences of patients with an implantable cardioverter defibrillator. Rehabil Nurs 1998;23:30-6

29 Vlay SC, Olson LC, Fricchione L, et al. Anxiety and anger in patients with ventricular tachyarrhythmias. Responses after automatic internal cardioverter defibrillator implantation. PACE 1989;12:366-73.

30 Bourke JP, Turkington D, Thomas G, et al. Florid psychopathology in patients receiving shocks from implanted cardioverter-defibrillators. Heart 1997;78:581-3.

31 Kohn CS, Petrucci RV, Baessler C, et al. The effect of psychological intervention on patients' long-term adjustment to the ICD: a prospective study. PACE 2000;23:450-6.

32 Badger JM, Morris PLP. Observations of a support group for automatic cardioverter defibrillator recipients and their spouses. Heart lung 1987; 18:238-43

33 Molchany CA, Peterson KA. The psychosocial effects of support group intervention on AICD recipients and their significant others. Prog Cardiovasc Nurs 1994;9:23-9.

34 Sneed NV, Finch NJ, Michel Y, et al. The effect of psychosocial nursing intervention on the mood state of patients with implantable cardioverter defibrillators and their caregivers. Prog Cardiovasc Nurs 1997;12:4-14. 